\title{
Interagency partnership to deliver Veteran-Directed Home and Community-Based Services: Interviews with Aging and Disability Network agency personnel regarding their experience with partner Department of Veterans Affairs medical centers
}

\author{
Kali S. Thomas, PhD, MA; ${ }^{*}$ Susan M. Allen, PhD \\ Center of Innovation in Long-Term Services and Supports for Vulnerable Veterans, Providence Department of Veterans \\ Affairs Medical Center, Providence, RI; Center for Gerontology and Health Care Research, Brown University, Provi- \\ dence, $R I$
}

\begin{abstract}
Veteran-Directed Home and Community-Based Services (VD-HCBS) is a consumer-directed program that began in 2009 and is jointly administered in a partnership between the Veterans Health Administration and the Administration for Community Living. The objective of this article is to describe the Aging and Disability Network agency (ADNA) personnel's perceptions of the implementation of the VDHCBS program with partner Department of Veterans Affairs medical centers (VAMCs). Qualitative interviews with 26 ADNA VD-HCBS personnel across the country were transcribed, coded, and analyzed. Results suggest that the majority of ADNA personnel interviewed perceive the collaboration experience to be positive. Interviewees reported several key mechanisms for facilitating a successful partnership, including frequent communication, training in VAMC billing procedures, having a designated VAMC staff person for the program, and active involvement of the VAMC from the onset of VD-HCBS program development. Findings have implications for other interagency partnerships formed to deliver services to vulnerable Veterans.
\end{abstract}

Key words: aging network, geriatrics and extended care, home and community-based services, implementation, interagency collaboration, interviews, long-term care, participant directed services, qualitative research, Veterans.

\section{INTRODUCTION}

In the past several decades, collaborations among federal agencies have become more common with the realization that the complex, crosscutting nature of challenging issues are best addressed by multiple agencies with overlapping and complementary responsibilities [1]. This is particularly true with efforts to improve community health. Notable examples of such partnerships are the successful collaboration of multiple federal agencies to achieve Healthy People 2010 objectives [2] and the partnership between the Veterans Health Administration (VHA) and the Department of Housing and Urban

Abbreviations: $\mathrm{ACL}=$ Administration for Community Living, ADNA = Aging and Disability Network agency, NRCPDS = National Resource Center for Participant-Directed Services, VA $=$ Department of Veterans Affairs, VAMC $=$ Department of Veterans Affairs medical center, VD-HCBS = VeteranDirected Home and Community-Based Services, VHA = Veterans Health Administration.

*Address all correspondence to Kali S. Thomas, PhD, MA; 830 Chalkstone Ave, Providence, RI 02908; 401-273-7100, ext 6268. Email: Kali.Thomas@va.gov

http://dx.doi.org/10.1682/JRRD.2015.02.0019 
Development, in which nearly 31,000 previously homeless Veterans have been moved into housing [3]. Such collaborations demonstrate the potential to pursue common goals in an efficient fashion by reducing duplication and fragmentation of efforts and by pooling multiple agency resources. However, there are also challenges to interagency collaborations associated with differing organizational cultures, lack of clarity in roles and responsibilities, inadequate commitment by leadership, and myriad other factors that may pose barriers to the implementation and effective functioning of these partnerships [4-6].

In this article, we focus on the partnership of two Federal agencies, the Administration for Community Living (ACL) and the VHA, formed to implement the Veteran-Directed Home and Community-Based Services (VD-HCBS) program through collaboration between ACL's Aging and Disability Network agencies (ADNAs) and the Department of Veterans Affairs (VA) medical centers (VAMCs) located in the same geographic area. This article describes ADNA personnel's perceptions of the implementation of the VD-HCBS program with partner VAMCs. Findings are discussed in the context of previous work identifying mechanisms that are liable to enhance the likelihood of federal interagency partnership success in operating across agency boundaries [7].

The Millennium Act of 1999 called for a rebalancing of long-term care in the VA, with decreased reliance on institutions and the expansion of home and communitybased services designed to support Veterans in their own homes or in the least restrictive setting possible [8]. Since that time, VHA Geriatrics and Extended Care Services has implemented innovative models of home-based care that seek to delay or avoid institutionalization altogether [9-10]. Recognizing the success of Medicaid's Cash and Counseling Program [11-12], Geriatrics and Extended Care Services leadership wanted to implement this model of self-directed home-based care for Veterans who require an extensive amount of disability-related personal assistance to maintain independent community living. In 2008, a partnership was formed between the VHA and ACL to implement the VD-HCBS program, essentially capitalizing on the ADNAs' home care service delivery experience and skills and the VHA's commitment and resources. Through this newly formed partnership, VAMCs purchase VD-HCBS on behalf of Veterans from State Units on Aging, Area Agencies on Aging, and Aging and Disability Resource Centers, known collectively as ADNAs.
Like the Cash and Counseling Program model [13], VD-HCBS provides Veterans with a monthly allotment based on an assessment of the extent of their need for assistance with personal care because of injury or the effect of disease. Veterans have the opportunity to spend their allotment on services that best meet their needs, such as personal care workers whom they hire and manage themselves, thus maximizing the concept of patientcentered care. Personal care workers may be family members who otherwise may not be able to afford to stay out of the workforce to care for a loved one. Allotted funds may also be spent on supplies, home modifications, and adaptive equipment.

The VAMCs are responsible for identifying Veterans who meet eligibility criteria for the VD-HCBS program, establishing their monthly budget based on their need, and then referring them to the ADNA. An ADNA representative then works with the Veteran to (1) develop a person-centered plan for the Veteran's care, (2) identify risks to Veterans' health and safety as well as how Veterans and their caregivers can mitigate those risks, (3) monitor spending and service utilization, and (4) provide ongoing counseling and support options. In addition, the Veteran (or appointed employer for services) and the ADNA representative work closely with a financial management service to manage employment taxes and insurance and process payroll and timesheets. The ADNA bills the VAMC for its assistance and oversight of VDHCBS recipients.

In an evaluation of the program conducted by Boston College's National Resource Center for ParticipantDirected Services (NRCPDS), survey responses from VAMC VD-HCBS coordinators indicated that VDHCBS is filling a special niche for Veterans at risk of nursing home placement [14]. The survey results further pointed out that for many, collaboration between VAMCs and ADNAs has been enhanced by mutual benefit; however, some VAMC VD-HCBS coordinators described these partnerships as complicated and difficult. The evaluation report suggests that in some cases, role clarification and coordination efforts may be helpful to maximize the efficiency of complementary roles of the VA and ADNA in education and outreach, assessment, matching Veterans' needs with program options and preferences, development of the budget and plan of care, and responsibilities for monitoring and follow-up. While the VAMC VD-HCBS coordinators generally rated the program as successful, the experience of participating ADNAs in 
working with their VAMC partners is not well documented. Therefore, the current study was designed to more completely understand the barriers and facilitators to the success of the VD-HCBS program implementation. Together, the reported experiences of both VAMC and ADNA personnel will be helpful at both the Federal and local levels in planning and implementation of future partnerships between community-based agencies and VAMCs for the purpose of delivering care to vulnerable Veterans.

\section{METHODS}

Twenty-six semistructured interviews were conducted with VD-HCBS ADNA representatives and analyzed using methods of qualitative analysis.

\section{Participants and Setting}

In May 2013, there were 43 VAMCs operating VDHCBS programs. Program leaders at the ACL provided the study team with contacts for all ADNAs that were currently administering the VD-HCBS program. We sent introductory letters to 33 individuals listed as contacts for the ADNAs. The letter described the study and indicated that a researcher would call to schedule the interview. Of these, 29 individuals were reached by telephone and 26 agreed to participate. The participants in this study included directors, project coordinators, and case managers from 18 Area Agencies on Aging, 7 Aging and Disability Resource Centers, and 1 State Unit on Aging who combined worked with 18 different VAMCs. Of these participants, all but four had been involved with the delivery of VD-HCBS from their program's inception.

\section{Data Collection}

Introductory letters were sent to the ADNA representatives inviting them to participate in individual interviews concerning the "potential benefits and drawbacks of the Veteran-Directed Home and Community Based Services program from the Aging and Disability Network Agency perspective." All interviews were conducted over the telephone and were carried out by two experienced interviewers from the VA.

Initially, we developed a semistructured interview guide with questions that addressed the topics of VDHCBS program benefits to Veterans and caregivers, experiences with program implementation, advice for other ADNAs considering partnering with VAMCs, and practices and policies surrounding program data collection and reporting. The Office of Performance and Evaluation at ACL and Boston College's NRCPDS then reviewed the interview guide. Changes that resulted from that review included additional questions and alternative wording for clarification to receive the best information possible.

We informed all participants that they had the right to withdraw from the study at any time. Each interview lasted approximately $35 \mathrm{~min}$ (range 18-63 $\mathrm{min}$ ) and was recorded, transcribed verbatim, and entered into the qualitative software program, NVivo (QSR International Pty Ltd; Melbourne, Australia). We checked the validity of the transcripts by parallel listening and reading through one-third of the 26 interviews.

\section{Data Analysis}

Four multidisciplinary team members (a gerontologist, health services researcher, sociologist, and public health researcher) with experience conducting qualitative research reviewed the transcripts independently. Each transcript was assigned to a minimum of two readers. As a group, we developed a codebook that we further refined by team consensus to reflect the content that the interview material elicited. We agreed on clearly defined codes prior to final coding, in which all previously coded transcripts were recoded for consistency using the finalized codes. Transcripts were reviewed at twice-monthly meetings, and team members shared the codes that they assigned to each segment of text. Whenever discrepancies between coders appeared, they were discussed and codes were finalized when a team consensus was reached. During this meeting, the team shared their general impressions of the findings. We kept an audit trail of coding decisions throughout the study.

As discussed previously, our semistructured interview guide included questions regarding the VD-HCBS program and Veteran recipients. However, because our focus in this article is on ADNA program personnel's perceptions regarding facilitators and barriers to coordination with VAMCs in delivering VD-HCBS, we report on participant responses to the question "Could you please describe your agency's overall experience working with the VD-HCBS program?” Respondents were not probed regarding specific areas of program implementation or collaboration with partner VAMCs. This grounded theory [15-16] approach to data collection and analysis 
was adopted to determine if the facilitators and barriers to implementation reported by VAMC personnel were mirrored in ADNA's perceptions or if different issues were raised. Responses to the question concerning overall experience working with the VD-HCBS program were initially coded to reflect either positive or negative reports of collaboration. More specific codes emerged directly from the data and were added as we observed recurring themes.

\section{RESULTS}

Overall, the majority of participants $(n=17 / 26)$ noted positive experiences with their involvement in the VAMC/ADNA VD-HCBS program partnership. Three additional individuals indicated that everything about their collaboration was positive except for aspects related to billing for ADNA services. One participant indicated that he/she had a positive experience until there was a leadership change at the VAMC, and two participants suggested that their program had evolved and that now the partnership is a positive collaboration. Three participants had predominately negative reports of their collaboration efforts with their local VAMCs.

Four themes were identified to exemplify mechanisms that facilitated a positive experience with the VDHCBS program: (1) frequent and effective communication between VAMC and ADNA VD-HCBS program personnel; (2) VAMC training in the policies and procedures for billing for ADNA services; (3) working with a VAMC-designated partner who served as champion for the program; and (4) VAMC involvement in all aspects of the ADNA's development of the program from the beginning, including the ADNAs' required readiness review.

The respondents who reported a negative experience with partnering VAMCs essentially reported the absence of the mechanisms listed, with the following results: (1) lack of sufficient VAMC staff time dedicated to the functioning of the VD-HCBS program, resulting in poor communication; (2) poor communication, resulting in delays in receiving VAMC approval for Veterans' care plans; and (3) ADNA's lack of understanding of VAMC billing procedures, resulting in ADNA payment delays. (They are paid for the oversight, monitoring, and fiscal management that they provide.)

Finally, regardless of the nature of the ADNAVAMC partnership, one or more funding issues, includ- ing inadequate funding allotted by the VAMC to the program to allow for growth, cuts in funding once the program was established, and uncertainty regarding continuity in funding, were reported by virtually all participants.

These themes are discussed subsequently along with illustrative quotes from survey respondents.

\section{Elements of a Successful Interagency Partnership in Delivering Veteran-Directed Home and Community- Based Services}

Many respondents cited effective communication between the partnering agencies as a key element of a positive working experience with VAMCs. In most instances, participants indicated that communicating about potential challenges from the outset of the partnership was one reason that they were able to avoid the frustration of their lack of experience with the protocols and procedures of a VAMC bureaucracy. As one respondent noted:

There was a lag time of three months when we weren't paid for anything; but we knew that in advance, that was part of our discussion of the realities of this, the VA system in payment is very slow; so we would just have to deal with it and it would be all right. That was part of our discussion before we ever signed a contract.

While effective communication early in the implementation process was clearly essential to a successful partnership, one participant stressed the need for frequent, ongoing communication in a partnership in which the tasks of enrolling, assessing, assisting, and monitoring Veterans are divided between two agencies: "I think it's working very well . . . we talk if not daily, multiple times per week, even if it's just for a couple of minutes. . . . I think without that we wouldn't have the program anymore."

To expedite smooth transactions between ADNAs and VAMCs, some ADNAs received specific training in VAMC policies and procedures for billing for ADNA services: "We were able to get some protocols in place . . . they spent some time giving us the training and information that we needed to accurately bill for services."

A designated VAMC representative dedicated to the VD-HCBS program at the partnering VAMC was also noted as a facilitator of effective functioning of the partnership. As one ADNA respondent explained: 
We had a wonderful working relationship with our local VA. We had one contact who is still our contact there and he has been totally on board and in support of this program ... when there is an issue, we can always call and get immediate response.

Another referred to VAMC “champions” for the program; that is, personnel who believed in the program's value to Veterans and were invested in making it work.

I think that a large factor in the program being able to be started and to be able to continue is we have a lot of champions for the program. There are champions here in our agency, there are champions at the VA . . . we have a lot of champions for the program ... that's probably the biggest thing.

An overarching theme voiced by several ADNA personnel that encompassed the themes discussed previously is the benefit of VAMC involvement with the ADNA during the development phase of the program:

They [the VA] did go out of their way to make sure they were coming when we were initially setting it up . . . people would attend these meetings which we knew was a commitment on their end. It really showed that they were committed to the program and making sure that we were really collaborating and it was a good partnership.

\section{Challenges of the Interagency Partnership}

The ADNA respondents who reported a negative experience in collaborating with VAMCs to implement the VD-HCBS program cited poor communication between the VHA at the national level and VAMCs regarding the VD-HCBS program itself, resulting in a poorly functioning partnership at the local level:

I think lack of communication of how the program runs; things from the Central Office, they go down to the local VA . . explaining the program, how it is, how it works. . . . It was just the lack of communication and people not being educated on the local level about the program. It made it hard to get things accomplished.

Not surprisingly, participants with negative experiences also had difficulty communicating with their partner at the local level. For example, one participant reported that the VA VD-HCBS coordinator at the local VAMC was "swamped with work" and this made com- munication difficult. Another reported frustration because some VAMCs do not communicate changes to Veterans' statuses in the program: "Sometimes the local VA will simply terminate [Veterans'] eligibility and we don't know why that is. Or, they'll just send us a letter and say he's no longer eligible and you can't serve him any longer."

In addition to poor communication between the ADNA and VAMC partners, frustrations were voiced about the time it took to get approvals and initiate service for the Veterans. As one respondent reported:

In the beginning all plans of care had to go through one individual person and it was one person who was extremely busy. It was a source of frustration for us managing the program because we couldn't begin services until the plans of care were approved and we couldn't really push any harder to get the plan of care approved. So there was a delay and we felt beholden to the Veteran and their family while we waited for these approvals.

Participants most frequently cited issues related to billing and receiving payment as the source of perceived negative collaboration with VAMCs; these issues resulted in an ADNA operating at a deficit for the program and delayed payments to its financial management service. One respondent expressed a common sentiment:

The cash flow is a challenge. It's a real challenge. So our [financial management service] is threatening to drop out of the whole program if the VA won't move to some other reimbursement system like giving us the funds a month ahead based on the budgeted amount.

Finally, the majority of participants involved in this study expressed frustration regarding funding allocated to the VD-HCBS program, even among those who otherwise reported positive aspects of collaboration with their partners. Some participants reported that the funding had not grown to meet the demand for the program. For example, one ADNA noted, "We have Veterans identified, we're ready to serve them-it's funding that's preventing us from being able to do that." Others expressed concern with the uncertainty of the budget being maintained for the program in the coming year. As one participant feared, “We really don't even know at this point whether or not they will continue with the program. It seems like it's year to year." 


\section{DISCUSSION}

Our findings confirm those of the earlier VD-HCBS program evaluation report [14]: the quality of the ADNA-VAMC partnership varies by site, with the majority of respondents reporting a positive collaborative experience. The ADNA respondents in our study who reported negative experiences cited poor communication between collaborating agencies, lack of training in VAMC billing procedures, and lack of a VAMC contact person who had the time and commitment required by the demands of the VD-HCBS program.

Reports of several of our respondents suggest that mutual participation from the outset of partnership, including during the ADNA's readiness review, can greatly facilitate development of positive working relationships that bridge organizational cultures, as can the development of procedures and strategies to minimize the challenges presented when operating across agency boundaries. While working together during the readiness reviews enabled ADNAs to become familiar with VAMCs' billing procedures and enabled VAMCs to become familiar with the workings of the VD-HCBS program itself, there are other mechanisms that may have been useful for the ADNA-VAMC partnership, including colocation of agency personnel or regional program offices that respond to the needs of both interagency partners [7].

A difficulty reported by all ADNA personnel relates to the uncertainty of a reliable funding stream for the VD-HCBS program. At the time of our study, VAMCs interested in offering this program received special purpose, time-limited funding from the VHA specifically for VD-HCBS program implementation. Participating ADNAs thus lacked the financial security warranted by the time, energy, and resources spent in program development. Fortunately, the Veterans Access, Choice, and Accountability Act of 2014 centralized funding allocations for purchased non-VA care services. As a result, funds are to be allocated to all VAMCs from the VA central office, and allocations explicitly include funding for VD-HCBS. Thus, ADNAs can now enter into partnerships with VAMCs with a guaranteed funding stream [17].

Though not reported in this article, participants unanimously noted many benefits of VD-HCBS program participation for the Veteran population it serves, including allowing the Veteran autonomy in determining the services that will best meet his or her needs and residence in the least restrictive setting. Such glowing reports mirror those reported in the Cash and Counseling Program demonstration and evaluation [11-12] and in the NRCPDS VD-HCBS program evaluation [14].

As the aging Veteran population continues to grow, the VHA recognizes the benefits of collaboration with other partners to meet Veterans' service needs. In addition, with the Veterans Access, Choice, and Accountability Act of 2014, the VA now contracts with providers to expand access to community healthcare when the VA is unable to provide timely care to Veterans who meet eligibility criteria. Therefore, the findings from this study have implications for future VA-community partnerships and can be informative for the organizations providing this contracting service as well as for the providers enrolling in the network.

It is important to note this study's limitations. One limitation is that the perspective in this study is limited to ADNA employees at organizations that were able to establish and operate a VD-HCBS program. We did not include any interviews with individuals who worked at ADNAs that were unable to launch a VD-HCBS program because of lack of interest by the VAMC or other issues that prevented its development. Additionally, the goal of this study was to understand the perceptions of the program from the ADNAs' perspective. Therefore, these findings do not include the voices of Veterans, caregivers, or national program leaders. Future work should incorporate interviews with Veterans and leadership to provide a holistic view of the program's many features.

\section{CONCLUSIONS}

In conclusion, our study finds that reports of the collaboration with VAMCs in delivering the VD-HCBS program vary and that there are collaborative mechanisms that can enhance the possibility for the partnerships' success. However, regardless of the quality of collaboration between the VAMCs and the ADNAs, participants unanimously note positive and beneficial outcomes of the delivery of VD-HCBS to Veterans and their caregivers. As the VA looks to serve more aging and disabled Veterans, it is important that collaborative relationships are mutually beneficial to both partners and ultimately improve the quality of and access to care for the Veteran population. 


\section{ACKNOWLEDGMENTS}

\section{Author Contributions:}

Study concept and design: K. S. Thomas, S. M. Allen.

Acquisition of data: K. S. Thomas.

Analysis and interpretation of data: K. S. Thomas, S. M. Allen.

Drafting of manuscript: K. S. Thomas, S. M. Allen.

Critical revision of manuscript for important intellectual content:

K. S. Thomas, S. M. Allen.

Statistical analysis: K. S. Thomas, S. M. Allen.

Study supervision: S. M. Allen.

Financial Disclosures: The authors have declared that no competing interests exist.

Funding/Support: This material was based on work supported by the Providence VA Medical Center Health Services Research \& Development Research Enhancement Award Program and Center of Innovation for Long-Term Services and Supports and the Agency for Healthcare Research and Quality (award T32 HS-000011) and by a Veterans Integrated Service Network 1 Career Development Award (award V1CDA2014-27).

Additional Contributions: We would like to acknowledge Deborah Burton, MA; Joshua Shreeve, MPH; and Gregory Boyer, PhD, for their assistance with interviews, coding, and data analysis. We would also like to thank the NRCPDS and the ACL for their assistance and guidance.

Institutional Review: The Providence VA Medical Center Institutional Review Board approved the study. All participants signed informed consent forms prior to the interview.

Participant Follow-Up: The authors plan to notify study subjects of the publication of this article.

\section{REFERENCES}

1. U.S. Government Accountability Office. Results-oriented government: Practices that can help enhance and sustain collaboration among Federal agencies. Washington (DC): GAO; 2005. GAO-06-15.

2. National Center for Health Statistics. Healthy People 2010: Final review. Hyattsville (MD): U.S. Department of Health and Human Services; 2012.

3. U.S. Government Accountability Office. Veteran homelessness: VA and HUD are working to improve data on supportive housing program. Washington (DC): GAO; 2012. GAO-12-726.

4. Lasker RD, Weiss ES, Miller R. Partnership synergy: A practical framework for studying and strengthening the collaborative advantage. Milbank Q. 2001;79(2):179-205, III-IV. [PMID:11439464] http://dx.doi.org/10.1111/1468-0009.00203

5. Butterfoss FD, Goodman RM, Wandersman A. Community coalitions for prevention and health promotion: Factors predicting satisfaction, participation, and planning. Health Educ Q. 1996;23(1):65-79. [PMID:8822402] http://dx.doi.org/10.1177/109019819602300105
6. Lasker RD, Weiss ES. Creating partnership synergy: The critical role of community stakeholders. J Health Hum Serv Adm. 2003;26(1):119-39. [PMID:15330382]

7. U.S. Government Accountability Office. Managing for results: Key considerations for implementing interagency collaborative mechanisms. Washington (DC): GAO; 2012. GAO-12-1022.

8. Veterans Millennium Health Care and Benefits Act of 1999, Pub. L. No 106-117, 113 Stat. 1545 (Nov. 30, 1999).

9. Edes T. Innovations in homecare: VA home-based primary care. Generations. 2010;34:29-34.

10. Shay K, Yoshikawa TT. Overview of VA healthcare for older veterans: Lessons learned and policy implications. Generations. 2010;34:21-28.

11. Carlson BL, Foster L, Dale SB, Brown R. Effects of cash and counseling on personal care and well-being. Health Serv Res. 2007;42(1 Pt 2):467-87. [PMID:1724493] http://dx.doi.org/10.1111/j.1475-6773.2006.00673.x

12. Simon-Rusinowitz L, Loughlin DM, Ruben K, Garcia GM, Mahoney KJ. The benefits of consumer-directed services for elders and their caregivers in the cash and counseling demonstration and evaluation. Public Policy Aging Rep. 2010;20:27-31. http://dx.doi.org/10.1093/ppar/20.1.27

13. Doty P, Mahoney KJ, Simon-Rusinowitz L. Designing the cash and counseling demonstration and evaluation. Health Serv Res. 2007;42(1p2):378-96. [PMID:17244289] http://dx.doi.org/10.1111/j.1475-6773.2006.00678.x

14. Mahoney E, Kayala D. Veteran-Directed Home and Community-Based Services: A program evaluation. Boston (MA): Trustees of Boston College, National Resource Center for Participant-Directed Services; 2013.

15. Glaser BG, Strauss AL. The discovery of grounded theory: Strategies for qualitative research. Chicago (IL): Aldine Publishing Company; 1967.

16. Strauss A, Corbin J. Basics of qualitative research: Grounded theory procedures and technique. 2nd ed. Newbury Park (CA): Sage; 1998.

17. National Resource Center for Participant Directed Services. VD-HCBS update: Progress in 2014 for VeteranDirected Home and Community-Based Services [Internet]. Boston (MA): Boston College; 2015 [cited 2015 Aug 15, updated $2015 \mathrm{Feb} 4$ ]. Available from: http://www.bc.edu/ schools/gssw/nrcpds/news/2014/vdhcbsprogress.html

Submitted for publication February 1, 2015. Accepted in revised form October 21, 2015.

This article and any supplementary material should be cited as follows:

Thomas KS, Allen SM. Interagency partnership to deliver Veteran-Directed Home and Community-Based Services: Interviews with Aging and Disability Network 
JRRD, Volume 53, Number 5, 2016

agency personnel regarding their experience with partner Department of Veterans Affairs medical centers. J Rehabil Res Dev. 2016;53(5):611-18.

http://dx.doi.org/10.1682/JRRD.2015.02.0019
ORCID: Kali S. Thomas, PhD, MA: 0000-0003-34362184

\begin{tabular}{|c|c|}
\hline $\begin{array}{l}\text { Au sueussions screeneo ar. } \\
\checkmark \text { iThenticate. }\end{array}$ & HEMBER \\
\hline 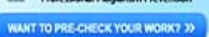 & $\begin{array}{l}\text { CROSSREF.ORG } \\
\text { THE CITATION LHIKING EACKRONE }\end{array}$ \\
\hline
\end{tabular}

